CONGENITAL HEART DISEASE

\title{
Inheritance of persistent foramen ovale and atrial septal defects and the relation to familial migraine with aura
}

\author{
P T Wilmshurst, M J Pearson, S Nightingale, K P Walsh, W L Morrison
}

Heart 2004;90:1315-1320. doi: 10.1136/hrt.2003.025700

See end of article for authors' affiliations .....................

Correspondence to: Dr Peter Wilmshurst, The Royal Shrewsbury Hospital, Shrewsbury SY3 8XQ, UK; peter.wilmshurst@ rsh.nhs.uk

Accepted 25 March 2004

\begin{abstract}
Objective: To determine whether smaller atrial shunts (large persistent foramen ovale (PFO) and small atrial septal defect (ASD)) are inherited and whether this has a role in the inheritance of migraine with aura.

Methods: Contrast echocardiography was used to detect atrial shunts in 71 relatives of 20 probands with a significantly sized atrial shunt (large PFO or ASD). Four families with three generations, 14 families with two generations, and two sibships were studied. The contrast echocardiograms were performed blind to history of migraine. A consultant neurologist, who was blinded to cardiac findings, categorised migraine symptoms in subjects.

Results: The occurrence of atrial shunts was consistent with autosomal dominant inheritance. Usually shunts were large PFOs, but in some cases they were ASDs. There was also evidence that inheritance of more complex congenital heart disease may be related to the inheritance of PFOs. When the proband had migraine with aura and an atrial shunt, 15 of the $21(71.4 \%)$ first degree relatives with a significant right to left shunt also had migraine with aura compared with three of 14 (21.4\%) without a significant shunt ( $\mathrm{p}<0.02)$.

Conclusions: There is dominant inheritance of atrial shunts. This is linked to inheritance of migraine with aura in some families.
\end{abstract}

$\mathrm{T}$ here is an association between migraine with aura and ight to left shunts. ${ }^{1-3}$ These shunts are usually across a large persistent foramen ovale (PFO) (9-16 mm diameter). ${ }^{4}$ Occasionally the shunt is through a small atrial septal defect (ASD) or a pulmonary arteriovenous shunt. ${ }^{4}$ Because even large PFOs do not have haemodynamic effects and cardiac examination is normal, their presence is not suspected unless there are other features to suggest a right to left shunt (such as paradoxical thromboembolism, decompression illness, or transient global amnesia). ${ }^{5-8}$

Some observations suggest that the association between such atrial shunts and migraine with aura is causative. Large shunts more often than small shunts are associated with migraine with aura. ${ }^{3}$ Paradoxical gas embolism across atrial shunts can precipitate migraine with aura and the severity of an episode of migraine appears to be related to the amount of bubbles crossing the defect. ${ }^{3}$ Closure of atrial shunts usually improves or cures migraine with aura. ${ }^{4}$

A family history is present in up to $90 \%$ of patients with migraine. ${ }^{9}$ Usually there is dominant inheritance with incomplete penetrance. ${ }^{9}$ There are few reports of families in which there was dominant inheritance of haemodynamically significant ASDs. ${ }^{10}$ We therefore investigated whether clinically silent atrial defects, such as large PFOs and small ASDs, are also inherited and whether this has a role in the inheritance of migraine.

\section{METHODS}

Transthoracic contrast echocardiography was used to investigate the prevalence of atrial shunts in members of 20 families (four families with three generations, 14 families with two generations, and two sibships). In 19 families the proband had a large atrial right to left shunt on contrast echocardiography. In the other family the proband had a large ASD detected by transoesophageal echocardiography alone. These shunts were found during investigation of shunt related decompression illness in 11 divers, ischaemic stroke resulting from paradoxical thromboembolism in three patients, haemodynamic features of an ASD in five patients (one of whom had only transoesophageal echocardiography), and severe migraine with aura in one patient. In those with decompression illness or stroke, other potential causes were excluded before attributing the illness to paradoxical embolism. Because of our study aim the proband in the majority of families we investigated had both an atrial shunt and migraine with aura (in 13 of 20 families studied).

Transthoracic contrast echocardiography was performed on 48 first degree relatives, 17 other blood relatives, and six relatives by marriage. In seven additional relatives information about congenital cardiac abnormalities was available. Twelve probands and two family members have had their atrial defect closed. PFOs with median diameter $14 \mathrm{~mm}$ (range 7-24 mm) at transcatheter closure were found in eight subjects, one of whom also had a small ASD closed. Two ASDs were closed with a transcatheter technique and each was $15 \mathrm{~mm}$ in diameter. Four subjects underwent surgical closure of larger ASDs whose precise dimensions were not recorded. Eight patients are waiting for a closure procedure. The study included two pairs of identical twins and one pair of non-identical twins.

Only individuals aged 18 years or older had contrast echocardiograms in this research study, but clinical information was available for some younger family members. Contrast echocardiography was performed blind to migraine history. The heart was imaged (apical four chamber view) with a Hewlett Packard Sonos echocardiographic machine. Bubble contrast was produced by pushing about $5 \mathrm{ml}$ sterile saline $(0.9 \% \mathrm{NaCl}), 0.5 \mathrm{ml}$ of the patient's blood, and $0.5 \mathrm{ml}$ air back and forth between two syringes connected by a three way tap until there were no visible bubbles. This mixture was injected through a 21 gauge butterfly needle into a left antecubital vein. The first contrast injection was performed

Abbreviations: ASD, atrial septal defect; PFO, persistent foramen ovale 
with the patient resting and breathing normally. If no shunt was seen with the first contrast injection or if there was any doubt whether the shunt was across an atrial shunt rather than a pulmonary arteriovenous shunt, up to five subsequent injections were performed with Valsalva manoeuvres with sudden release, as described previously. ${ }^{11}$ If no shunt was seen at that stage, further contrast echocardiography was not performed. If there was a right to left shunt, it was graded according to the maximum number of bubbles seen in the left heart on frame by frame analysis: small shunts were defined by fewer than six bubbles, medium shunts by six to 20 bubbles, and large shunts by more than 20 bubbles. ${ }^{5}$ We consider that small shunts are not clinically significant. ${ }^{12}$

In probands and relatives, migraine with aura or without aura was diagnosed according to the diagnostic criteria of the International Headache Society during a structured interview with a consultant neurologist. ${ }^{13}$ The neurologist was blinded to the cardiac history and findings, except in two individuals, whom he had previously seen to advise whether shunt closure was indicated. Three individuals could not be contacted for neurological review. For one of the three, sufficient information from the medical records allowed a headache classification. For the other two there was inadequate recorded medical information about migraine symptoms to allow diagnosis.

Groups were compared by the $\chi^{2}$ test. For $2 \times 2$ tables Yates's correction was used.
The Shropshire Research Ethics Committee approved the study and participants gave informed consent.

\section{RESULTS}

Family 1

Figure 1 shows the pedigree of family 1 . The proband had a 30 year history of migraine with aura. At the age of 46 she had an ischaemic stroke after a long haul flight soon after starting oestrogen treatment. No cause for her stroke was found other than a large right to left shunt at rest. A $24 \mathrm{~mm}$ diameter PFO was found at transcatheter closure. The defect was not an ASD because it was completely covered by a flap during part of the cardiac cycle. Twenty of this patient's blood relatives, including five first degree relatives and three people related by marriage, had contrast echocardiography. Her mother, sister, and daughter had large shunts at rest and migraine with aura. Her sister also had a history of transient global amnesia. One brother had a large shunt at rest and a 25 year history of migraine. His migraines were associated with some brief visual disturbances but these were not considered to be typical of migraine aura. A second brother had no shunt but had infrequent migraine with aura. In this family, 14 blood relatives of the proband had a right to left shunt (11 large, two medium, and one small) with features suggesting that the shunts were across a PFO. The pedigree suggests that there is dominant inheritance of atrial shunts in this family. There is evidence of father to son transmission

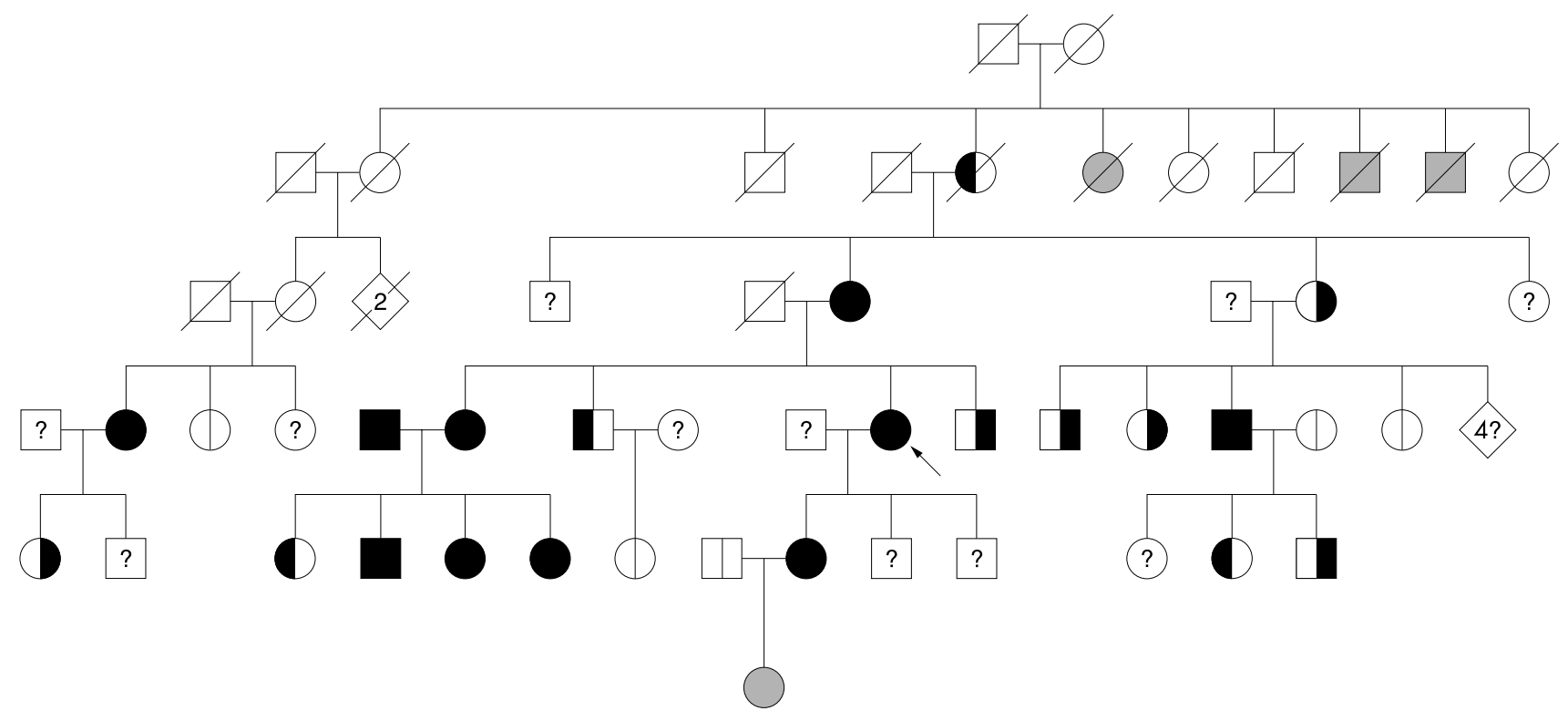

\begin{tabular}{|l} 
Male Atrial shunt and migraine with aura \\
Atrial shunt but no migraine with aura \\
Cyanotic congenital heart disease \\
Dead - no information about shunt or migraine
\end{tabular}

Figure 1 Family 1 pedigree. 
in a cousin of the proband. The husband of the sister of the proband also had a history of migraine and a large atrial shunt. The four offspring of that marriage had migraine with aura and three had a large atrial shunt, but one had no shunt. The granddaughter of the proband had surgical correction of truncus arteriosus, ventricular septal defect, and a PFO. The grandmother of the proband is dead. She is reported to have had severe migraine with visual disturbances, but we do not know whether she had a shunt. She had eight siblings, of whom a sister died at age 18 of cyanotic congenital heart disease and two brothers died in infancy of cyanotic congenital heart disease.

\section{Family 2}

Figure 2 shows the pedigree of family 2 . The proband is a diver who had two episodes of decompression illness (one neurological and one cutaneous). Her dive profiles and latency after surfacing are in keeping with shunt related decompression illness. She had numerous episodes of postdive migraine aura and a 25 year history of migraine with aura unrelated to diving. She had transcatheter closure of a $10 \mathrm{~mm}$ diameter PFO and a $4 \mathrm{~mm}$ ASD. Her sister, nephew, and mother had migraine with aura and each had a large right to left shunt with characteristics of a large PFO. Her mother also had a history of stroke at the age of 30 and transient global amnesia.

\section{Families 3 and 4}

The pedigrees of families 3 (fig 3 ) and 4 (fig 4) are consistent with dominant inheritance. In family 3 the proband had shunt related decompression illness and migraine with aura. During transcatheter closure he was found to have a $16 \mathrm{~mm}$ diameter PFO. Both of his sons, his mother, and one brother had large right to left shunts consistent with large PFOs. His other brother declined testing, but that brother's son is awaiting surgical closure of a secundum ASD; the defect is too large to close with a transcatheter technique.

\section{Two-generation families with identical twins}

Figure 5 shows the pedigree of family 5 . The proband had five episodes of shunt related decompression illness (one neurological and cutaneous and four cutaneous alone) and migraine with aura. He had a $14 \mathrm{~mm}$ diameter PFO at transcatheter closure. His identical twin had a $7 \mathrm{~mm}$ PFO closed, a single episode of cutaneous shunt related decompression illness, and frequent and severe migraine. Although the proband's twin had some visual disturbance during the headaches these were not considered to be aura. Their brother declined contrast echocardiography, but their mother

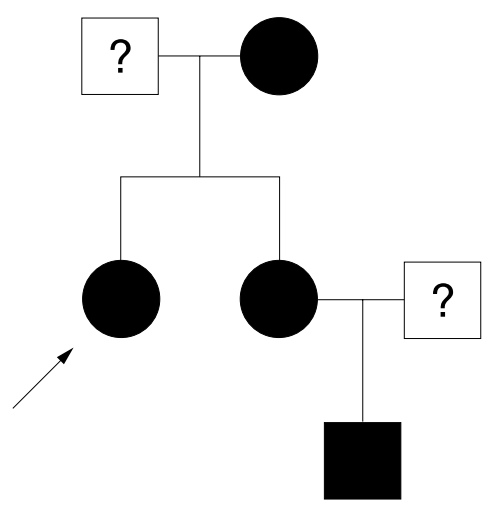

Figure 2 Family 2 pedigree. See fig 1 for key.

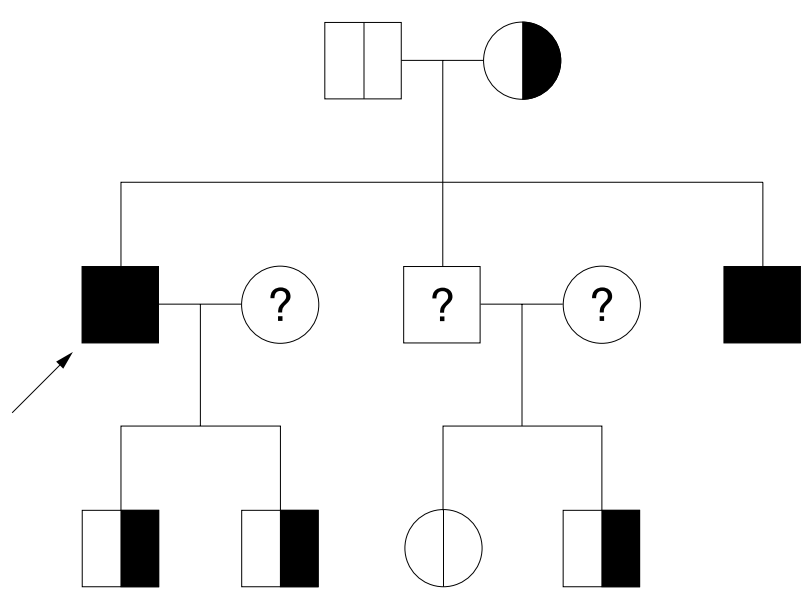

Figure 3 Family 3 pedigree. See fig 1 for key.

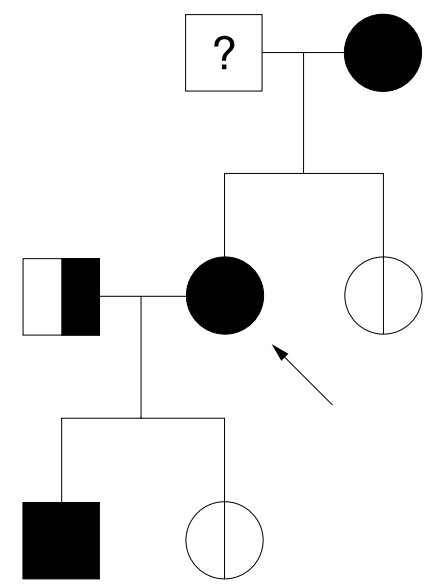

Figure 4 Family 4 pedigree. See fig 1 for key.

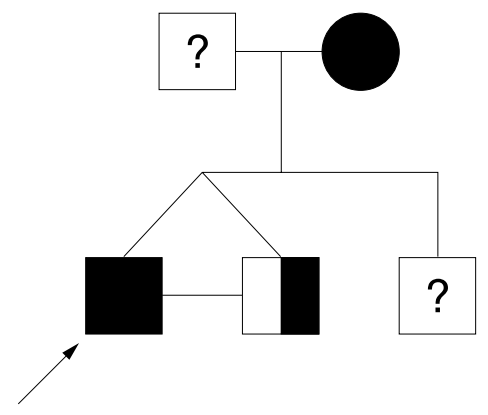

Figure 5 Family 5 pedigree. See fig 1 for key.

had migraine with aura and a large right to left shunt consistent with a PFO.

The pedigree of family 6 (fig 6) also included a pair of identical twins. Neither of them had a right to left shunt, but one had experienced four episodes of migraine with aura. Their mother, the proband, had a 40 year history of migraine with aura, with attacks currently occurring once or twice a week. She had neurological decompression illness and is awaiting transcatheter closure of a $15 \mathrm{~mm}$ diameter secundum ASD. Her clinical cardiac examination was normal. Her third daughter had migraine without aura and no shunt. Her son had a large right to left atrial shunt but no migraine. 


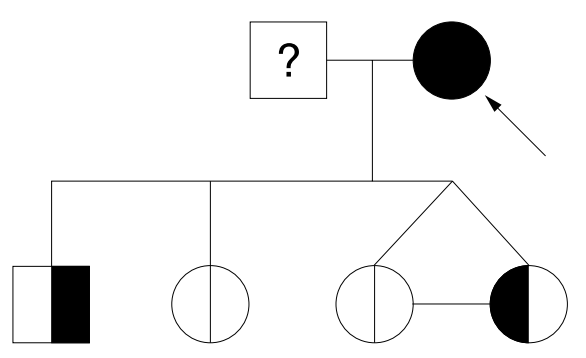

Figure 6 Family 6 pedigree. See fig 1 for key.

\section{Other two-generation families}

Twelve other two-generation families were studied. In four families both parents of the proband were investigated. In one family the mother had a right to left shunt and the father did not. In another only the father had a shunt. In another the father had a large shunt with the characteristics of a PFO and the mother had a medium sized shunt with characteristics of a pulmonary shunt. In the fourth family neither parent had a shunt. In the cases of two other probands, only one parent was available for investigation. In one case this was the father of the proband and in the other it was the mother, each of whom had a shunt. In six families, the children of the proband were investigated and six of 11 children were found to have a shunt. We identified four families in which a parent had a secundum ASD and one or more children had a PFO. We also identified a case where a mother had a PFO and her son had a secundum ASD. In other families there appeared to be father to son, father to daughter, mother to son, and mother to daughter transmission of atrial shunts. In three of these families a total of five sisters of the probands were also investigated. In one family the proband had a PFO closed and both her sisters had a right to left shunt in keeping with a PFO. In another family, in which the proband had a PFO, one of her sisters had severe migraine without aura and had a secundum ASD closed at age 13 years, but she did not have contrast echocardiography to see whether she had a residual shunt. Her other sister, who had migraine with aura, was found on transoesophageal echocardiography to have two ASDs $(11 \mathrm{~mm}$ and $4 \mathrm{~mm}$ diameters) and is awaiting transcatheter closure. In the last family the sister had no shunt.

\section{Sibships}

In a pair of non-identical twins, one sister had an atrial right to left shunt and had decompression illness. Her twin sister had been her diving companion at the time, but was not affected and did not have a shunt. We do not have details of migraine history in this pair of non-identical twins. In a pair of brothers, one had a sinus venosus ASD and the other had no shunt.

\section{Migraine}

Migraine with aura was diagnosed in 13 probands and severe migraine without aura in three. Three had no migraine. (Categorisation was not possible for one proband, who was one of the non-identical twins for whom details of migraine history was not available.)

When the proband had migraine with aura, 21 of the 35 first degree relatives we investigated had a significant (large or medium) right to left shunt. Fifteen of the 21 (71.4\%) with a significant right to left shunt also had migraine with aura, compared with three of $14(21.4 \%)$ without a significant right to left shunt $(\mathrm{p}<0.02)$. When the proband did not have migraine with aura, five of 11 first degree relatives had a right to left shunt on our contrast echocardiograms and two others were shown to have ASDs on transoesophageal echocardiography. Two of five (40\%) with a significant shunt or ASD had migraine with aura compared with four of eight (50\%) without a significant shunt (not significant).

Excluding the probands, we found that in these families 33 (46.5\%) of 71 family members who had contrast echocardiography had large shunts, five $(7.0 \%)$ had medium shunts, five $(7.0 \%)$ had small shunts, and $28(39.4 \%)$ had no shunt. For one subject information on migraine history was not available. Twenty one of $33(63.6 \%)$ with large shunts, four of $10(40 \%)$ with medium and small shunts, and eight of 27 $(29.6 \%)$ without shunts had migraine with aura. (The data from those with medium and small shunts were combined because these groups were small and an identical proportion in each group (two of the five) had migraine with aura.) The relation between size of shunt and prevalence of migraine with aura in family members (excluding probands) was significant $(\mathrm{p}<0.05)$.

\section{DISCUSSION}

A necropsy study of 965 hearts showed that $27.3 \%$ of adults had a PFO, but a defect $10 \mathrm{~mm}$ in diameter or larger was found in only $13(1.3 \%) .{ }^{14}$ We have previously reported that $27.6 \%$ of controls had a right to left shunt on transthoracic contrasts echocardiography, but only $4.9 \%$ had a large shunt without provocative manoeuvres and a further $2.4 \%$ had a large shunt with a Valsalva manoeuvre. ${ }^{12}$ People with shunt related clinical events, such as decompression illness and stroke due to paradoxical embolism, usually have the largest shunts on contrast echocardiography and these correspond to PFOs with median diameters of $11 \mathrm{~mm} .^{15}$

In this study a much higher proportion of the relatives of probands also had an atrial shunt and in particular a larger atrial shunt than would be expected in a normal population. This suggests that a large shunt in a proband predicts a higher than normal prevalence in relatives. There is one other study that investigated whether atrial shunts are familial. ${ }^{16}$ That study recruited a group of 62 patients who had an ischaemic stroke from a group of $308(20.1 \%)$ of those seen. The patients and a control sibling of the same sex and nearest in age were studied by contrast transcranial Doppler. Of the 62 patients recruited, 26 (nine male and 17 female patients) had a PFO; $76.5 \%$ of female siblings of patients with a PFO also had a PFO, but only one third of male siblings of men with a PFO also had a PFO. The authors concluded that "in women, PFO is a family trait". The study design is flawed because comparison of same sex siblings of similar age allows maximum influence of environmental factors. The findings cannot account for why a PFO is as frequent in men as in women. The observations do not fit any known pattern of mendelian or mitochondrial inheritance. The proof of inheritance of disease requires evidence of passage of disease or disease traits from generation to generation. Only the use of pedigrees with evidence of passage between generations allows one to determine whether the pattern of inheritance fulfils accepted criteria. It is clear that in the families we studied the pattern of occurrence of atrial shunts is consistent with dominant inheritance. Our observations in twins suggest that inheritance is more important than environment, but only three pairs were investigated.

In most affected people the defect was a large PFO, but in some there was a secundum ASD or both an ASD and PFO. Previous failure to recognise the clear pattern of inheritance of this condition may be because defects of this type are not associated with abnormal cardiac findings and because doctors do not usually recognise the symptoms associated with them; the most frequent symptom is migraine with aura. $^{1-3}$

It is well established that complex congenital heart disease has high prevalence in some families, but it does not appear 
to follow a mendelian pattern of inheritance. Family 1 with four members who had complex cyanotic congenital heart disease may provide an explanation. A possible connection between a child who had surgical correction of truncus arteriosus, ventricular septal defect, and a PFO and three relatives who were born four generations earlier and who also had cyanotic congenital heart disease are linking relatives who had atrial shunts and migraine with aura. Our data suggest that there may be dominant inheritance of a predisposition to cardiac abnormalities, which sometimes manifests as severe heart disease and sometimes only as an atrial shunt. In support of this, we have observed that in a number of other families in which the proband has a large PFO, there is a high incidence of other cardiac abnormalities, including ventricular septal defects, coarctation of the aorta, and patent ductus arteriosus (unpublished observations).

This study confirms our previous observation that the prevalence of migraine with aura is greatest in people with large shunts. ${ }^{3}$ The presence of a large right to left atrial shunt may explain the association of migraine with aura with both stroke and decompression illness. ${ }^{17}$ It is notable that among the non-probands we found some who had shunt related events other than migraine with aura, such as transient global amnesia, stroke at a young age, and decompression illness.

The number of people with migraine with aura in this study was large partly because we aimed at studying families in which either the proband or relatives of the proband had migraine with aura. We believe that people with migraine with aura were more likely to volunteer for the study than those without migraine. When the proband had migraine with aura, other family members who had migraine with aura were particularly keen to participate in the study and they usually had a large atrial shunt as well.

The association between migraine with aura and atrial shunts is not absolute, even in these families. There are several possible explanations for this. For example, our diagnosis of migraine with aura or of atrial shunts may be inaccurate. Migraine with aura is a clinical diagnosis and depends on accurate recollection and description of symptoms. In younger individuals, absence of migraine with aura does not mean that it will not occur later in life. It is commonly believed that transoesophageal echocardiography is the best method for detecting a PFO and that transthoracic contrast echocardiography is inferior. However, we have shown that our technique is better at detecting a clinically important PFO than when transoesophageal echocardiography is performed by an expert operator in the UK. ${ }^{18}$ Of course, contrast echocardiography may give false results on occasion, but we have a detection rate for atrial shunts in control subjects of $27.6 \%,{ }^{12}$ which is very close to the prevalence of PFO detected after death. ${ }^{14}$ We have performed transcatheter closure of atrial shunts in over 150 cases purely on the basis of our contrast echocardiogram without diagnostic transoesophageal echocardiography. In all cases except one, a large atrial shunt was confirmed at cardiac catheterisation; in the exceptional case, the patient had a pulmonary arteriovenous malformation. We are therefore confident that there are no major inaccuracies in our contrast echocardiography technique.

However, it is well established that the association between migraine with aura and atrial shunts is not perfect. ${ }^{1-3}$ Closure of atrial shunts cured migraine with aura in about half of those affected but only improved migraine with aura in the other half. ${ }^{4}$ These observations suggest that an atrial shunt merely facilitates migraine with aura. It may do so by permitting venous blood to bypass the lungs, where vasoactive chemicals and microemboli are filtered. In individuals who do not have a shunt, other mechanisms must be responsible for migraine with aura. It is possible that the amount of vasoactive chemical in venous blood in some people may be sufficient to overwhelm the capacity of the lungs to filter it or the lungs may be less efficient. In some the pathogenesis of migraine may be unrelated to bloodborne chemicals.

When the proband did not have migraine with aura, the pattern of atrial shunts in the families was still consistent with dominant inheritance, but there was no association between migraine with aura and the presence of a shunt. This suggests that in those families another mechanism was the main causative factor of migraine with aura.

The findings of this study show that large atrial shunts can be dominantly inherited and that they may be a phenotype marker for a gene responsible for the occurrence of more complex congenital heart disease in some families. These findings increase our understanding of pathophysiological mechanisms in migraine with aura, including its inheritance.

\section{APPENDIX}

Since this study was submitted for publication we have investigated five further probands with a PFO and 24 of their relatives (one three-generation family and four two-generation families). Each family has evidence of dominant inheritance of atrial shunts and in one family a woman with a large atrial shunt and migraine with aura has a daughter with tetralogy of Fallot and migraine with aura.

\section{ACKNOWLEDGEMENTS}

This research would not have been possible without the help of cardiorespiratory technicians at the Royal Shrewsbury Hospital. We are grateful to Mr Ian Roth, Department of Medical Illustration at the Royal Shrewsbury Hospital, and Dr Toni Harper, Department of Clinical Genetics, Birmingham Women's Hospital, for help with the pedigrees.

\section{Authors' affiliations}

P T Wilmshurst, M J Pearson, S Nightingale, Royal Shrewsbury Hospital, Shrewsbury, UK

K P Walsh, Our Lady's Hospital for Sick Children, Dublin 12, Ireland

W L Morrison, The Cardiothoracic Centre, Liverpool, UK

Conflict of interest: None

Contributions of authors: PW conceived the study, collected and analysed the data, and wrote the first draft of the paper. PW and MP performed the contrast echocardiograms. SN did the neurological assessment of patients. KW and WM performed cardiac catheterisation and transcatheter closure of atrial shunts. All authors amended the paper and approved the final version.

\section{REFERENCES}

1 Del Sette $M$, Angeli S, Leandri M, et al. Migraine with aura and right-to-left shunt on transcranial Doppler: a case-control study. Cerebrovasc Dis 1998:8:327-30.

2 Anzola GP, Magoni M, Guindani M, et al. Potential source of cerebral embolism in migraine with aura: a transcranial Doppler study. Neurology 1999;52:1622-5.

3 Wilmshurst P, Nightingale S. Relationship between migraine and cardiac and pulmonary right-to-left shunts. Clin Sci 2001;100:215-20.

4 Wilmshurst PT, Nightingale S, Walsh KP, et al. Effect on migraine of closure of cardiac right-to-left shunts to prevent recurrence of decompression illness or stroke or for haemodynamic reasons. Lancet 2000;356:1648-51.

5 Webster MWI, Chancellor AM, Smith $\mathrm{HJ}$, et al. Patent foramen ovale in young stroke patients. Lancet 1988;318:1144-52.

6 Moon RE, Camporesi EM, Kisslo JA. Patent foramen and decompression sickness in divers. Lancet 1989;i:513-4.

7 Wilmshurst PT, Byrne JC, Webb-Peploe MM. Relation between interatrial shunts and decompression sickness in divers. Lancet 1989;ii:1302-6.

8 Klotzsch C, Sliwka U, Berlit $P$, et al. An increased frequency of patent foramen ovale in patients with transient global amnesia: analysis of 53 consecutive patients. Arch Neurol 1996;53:504-8.

9 Bradley WG, Daroff RB, Fenichel GM, et al. Neurology in clinical practice, 3rd ed. Boston: Butterworth-Heinemann, 2000:1846.

10 Benson DW, Sharkey A, Fatkin D, et al. Reduced penetrance, variable expressivity, and genetic heterogeneity of familial atrial septal defects. Circulation 1998;97:2043-8. 
11 Wilmshurst P, Davidson C, O'Connell G, et al. Role of cardiorespiratory abnormalities, smoking and dive characteristics in the manifestations of neurological decompression illness. Clin Sci 1994;86:297-303.

12 Wilmshurst $\mathbf{P}$, Bryson P. Relationship between the clinical features of neurological decompression illness and its causes. Clin Sci 2000;99. 65-75.

13 Anon. Classification and diagnostic criteria for headache disorders, cranial neuralgias and facial pain. Headache Classification Committee of the International Headache Society. Cephalalgia 1988;8(suppl 7):1-96.

14 Hagen PT, Scholz DG, Edwards WD. Incidence and size of patent foramen ovale during the first 10 decades of life: an autopsy study of 965 normal hearts. Mayo Clin Proc 1984;59:17-20.
15 Wilmshurst $\mathbf{P}$. Foramen ovale permeable et plongee: quelle strategie? In: Communication Global Sante, eds. Coeur et plongee. Paris: Societe Francaise de Cardiologie, 2003:33-9.

16 Arquizan C, Coste J, Touboul P-J, et al. Is patent foramen ovale a family trait? A transcranial Doppler sonographic study. Stroke 2001;32:1563-6.

17 Engel GL, Webb JP, Ferris EB, et al. A migraine-like syndrome complicating decompression sickness. Scintillating scotomas, focal neurologic signs and headache: clinical and electroencephalographic observations. War Med 1944;5:304-14.

18 Wilmshurst PT, Pearson MJ, Walsh KP, et al. In clinical practice transoesophageal echocardiography usually fails to detect large persistent foramen ovale. Heart 2003;89:iii38-9.

\section{IMAGES IN CARDIOLOGY}

\section{Left ventricular diverticulum in a neonate with Cantrell syndrome}

A newborn baby was transferred to our hospital because of a pulsating mass of the abdominal wall just above the umbilical insertion. The patient was comfortable, not cyanotic and breathing spontaneously, but with mild tachypnoea. Physical examination was unremarkable except for a pulsating purple mass in the midline just above the umbilicus (middle panel). The chest $x$ ray showed a moderate dextroposition of the heart without any pulmonary pathology. The ECG was normal. Echocardiography revealed a normal intracardiac anatomy except for a long and narrow appendiform diverticulum at the apex of the left ventricle of almost $3 \mathrm{~cm}$, contracting synchronously with the left ventricle. The left superior caval vein persisted and entered into the right atrium via the coronary sinus.

Multisliced computed tomography showed a bifid lower part of the sternum, a small omphalocoele, a ventral diaphragmatic hernia, the absence of the apical part of the pericardial membrane, and the abnormal left ventricle with its diverticulum. The heart was noted to be in a dextroverse position. No other abnormalities were encountered.

The diagnosis of pentalogy of Cantrell consists of a combination of an abdominal wall defect, a diaphragmatic defect in the sternal part, a sternal defect, absence of the inferior pericardial membrane, and a heart defect, most frequently tetralogy of Fallot or a left ventricular diverticulum. The diagnostic results above confirmed our suspicion of this syndrome.

Surgical resection of the left ventricular diverticulum was indicated because of the risk of incarceration of the intestinal part in the diaphragmatic hernia. A preoperative cardiac catheterisation was performed revealing normal

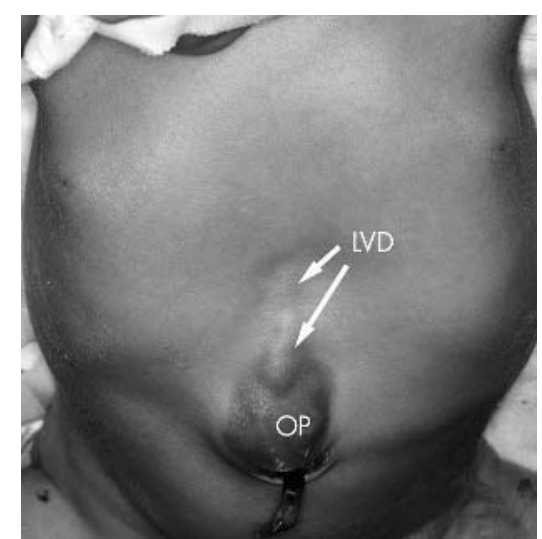

Thorax and abdomen demonstrating the abdominal mass on the midline, being the left ventricular diverticulum (LVD), as well as the small omphalocoele (OP).

intracardiac pressures and saturations. Angiography of the left ventricle showed the ventricle with the diverticulum and visualised a normal aorta with two normal coronary arteries (right upper panel). The right and left coronary artery systems were normally dispersed over the myocardium and no main branch descended into the wall of the diverticulum. of the left ventricular diverticulum (right lower panel) was performed and the apex of the left ventricle was closed, using two pledgets to prevent myocardial rupture. The abdominal wall was closed successfully after repair of the diaphragm in the same session at one week of age. At 3 weeks of age the patient was discharged in good general condition.

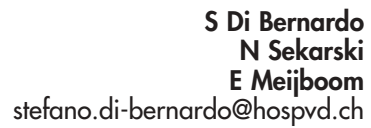

Based on this information a resection

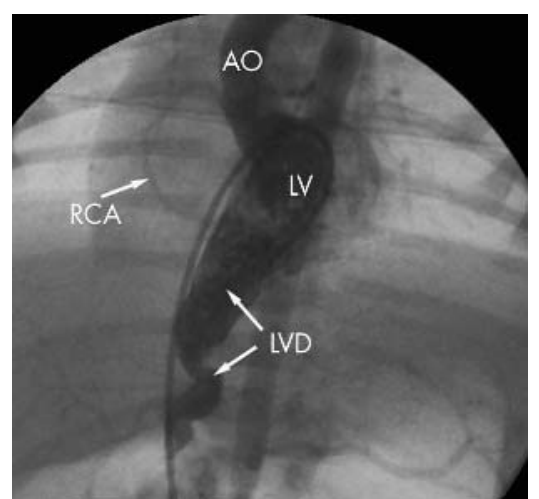

Angiography of the left ventricle (LV) with the catheter coming from the vena cava inferior through the foramen ovale, demonstrating the dextroverse position of the heart and the left ventricular diverticulum (LVD), passing through the diaphragm pointing at the umbilicus. RCA, right coronary artery; $\mathrm{AO}$, ascending aorta.

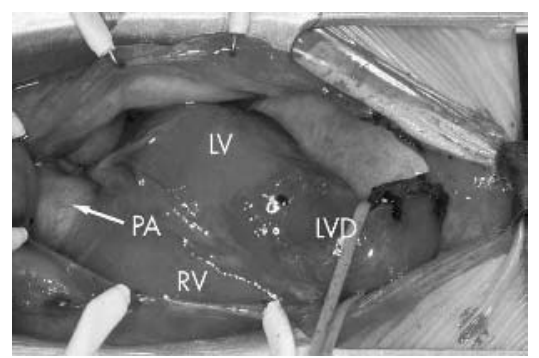

Anatomical view during surgery with a sternotomy and a midline laparotomy revealing the dextroverse position of the heart, and the left ventricular diverticulum (LVD) prolonging the left ventricle (LV) into the abdomen through the diaphragmatic hernia and terminating at the umbilicus in the omphalocoele. RV, right ventricle; PA, main pulmonary artery. 